\title{
Correction to: An Approach Toward the Economic Assessment of Business Process Compliance
}

Stephan Kuehnel and Andrea Zasada

\begin{abstract}
Correction to:
Chapter "An Approach Toward the Economic Assessment of Business Process Compliance" in: C. Woo et al. (Eds.): Advances in Conceptual Modeling, LNCS 11158, https://doi.org/10.1007/978-3-030-01391-2_28
\end{abstract}

In the original version of this chapter, the term "vector product" was used instead of "scalar product" in the first paragraph of page 236. This has now been corrected. 